\title{
Novel therapeutic applications of cannabinoids in cancer disease
}

\author{
Walter Milano ${ }^{1}$, Mario F Tecce ${ }^{1}$ and Anna Capasso ${ }^{2 *}$ \\ ${ }^{1}$ Mental Health Unit District 24 ASL Napoli 1 Center, Italy \\ ${ }^{2}$ Department of Pharmacy, University of Salerno, 84084-Fisciano, Italy
}

\begin{abstract}
The endocannabinoid system, comprising the cannabinoid receptors type 1 (CB1) and type 2 (CB2), their endogenous ligands (endocannabinoids), and the proteins that regulate endocannabinoid biosynthesis and degradation, controls several physiological and pathological functions. Indeed, recent evidence indicates that endocannabinoids influence the intracellular events controlling the proliferation and apoptosis of numerous types of cancer cells, thereby leading to both in vitro and in vivo antitumour effects. Also, the endogenous ligand arachidonoyl ethanolamide (anandamide; AEA) inhibits the proliferation of human breast cancer cells by blocking the G0/G1-S-phase transition of the cell cycle through interference with cannabinoid CB1 receptor-coupled signal-transducing events. The present review shows that cannabinoids exert their anti-cancer effects in a number of ways and in a variety of tissues. Furthermore, the novel therapeutic applications of cannabinoids in cancer disease, described here, strongly support the idea that cannabinoids may induce benefical effect in cancer treatment.
\end{abstract}

\section{Introduction}

The recreational use of Cannabis Sativa preparations is known to most people, largely as a result of the explosion in its use in the late 1960s; indeed, marijuana is still one of the most widespread illicit drugs of abuse in the world [1]. However, the medicinal use of Cannabis also has a millenarian history [2], although this history has been reexamined only very recently [3]. As early as $2600 \mathrm{BC}$, the Chinese emperor Huang Ti advised taking Cannabis for the relief of cramps, and rheumatic and menstrual pain; however, the great therapeutic potential of Cannabis was not scientifically assessed and publicized in the Western world until the British physician O'Shaugnessy wrote on the topic in the nineteenth century [2].

This long history of Cannabis use has resulted in the development of pharmaceutical drugs, such as dronabinol (Marinol;Unimed). This preparation, prescribed in the United States as an anti-emetic and appetite-stimulants to patients with cancer and AIDS, is based on $\Delta^{9}$-tetrahydrocannabinol (THC), which in 1964 by Mechoulam and coworkers was identified as the major psychoactive component of cannabis. To date, some 60 plant terpenophenols more or less related to THC have been isolated and defined cannabinoids [4]. Although the pharmacology of most of the cannabinoids is unknown, it is widely accepted that $\Delta^{9}$-tetrahydrocannabinol is the most important, owing to its high potency and abundance in cannabis.

\section{Cannabinoid receptors}

So far, two cannabinoid-specific receptors have been cloned and characterized from mammalian tissues, the seven transmembrane $G$ protein-coupled cannabinoid receptors type 1 (CB1 receptor), [5] and type 2 (CB2 receptor) [6]. Both the central effects and many of the peripheral effects of cannabinoids depend on $\mathrm{CB} 1$ receptor activation. Expression of this receptor is abundant in the brain, particularly in the basal ganglia, cerebellum and hippocampus, which accounts for the well-known effects of cannabis on motor coordination and short term memory processing [7]. The CB1 receptor is also expressed in peripheral nerve terminals and various extraneuronal sites such as the testis, eye, vascular endothelial and spleen. By contrast, the CB2 receptor is almost exclusively expressed in the immune system, both by cells, including B and T lymphocytes and macrophages, and by tissues, including the spleen, tonsils and lymph nodes [8-10]. Pharmacological evidence exists for the presence of other cannabinoid receptors, which, however, have not yet been cloned [11]. CB1 and CB2 receptors share only $44 \%$ overall identity and $68 \%$ within the transmembrane domains. Both cannabinoid receptors cloned so far are coupled to $G$ proteins, mostly of the $G_{i / o}$ type, through whose a subunit they modulate the activity of adenylate cyclases (inhibited) and mitogen-activated protein kinases (stimulated). CB1 receptors are also coupled to modulation of voltage-activated $\mathrm{Ca}^{2+}$ channels (inhibited) and inwardly rectifying $\mathrm{K}^{+}$ channels (stimulated), and activation of both phospholipase $\mathrm{C}$ (via the $\beta \gamma$ subunits of the G protein) and PI-3-kinase. CB2 receptors, on the other hand, trigger a sustained activation of ceramide biosynthesis [12].

\section{Cannabinoids and cancer}

The ubiquity of the endogenous ligands of cannabinoid receptors in both vertebrate and invertebrate tissues, and their modulating activity on proteins and nuclear factors involved in cell proliferation, differentiation and apoptosis, suggest that the endocannabinoid signalling system is involved, among other effects, in the control of cell survival transformation and proliferation. The anti-proliferative properties of cannabis compounds were first reported almost 30 years ago by Munson et al., who showed that THC inhibits lung-

Correspondence to: Anna Capasso, Department of Pharmacy, University of Salerno, 84084-Fisciano, Italy, E-mail: annacap@unisa.it

Key words: cannabinoid, cancer treatment, therapeutic application

Received: November 10, 2017; Accepted: December 12, 2017; Published: December 16,2017 
adenocarcinoma cell growth in vitro and in vivo. Although these observations were promising, further studies in this area were not carried out until the late 1990s, when the effects of plant-derived (for example, THC and cannabidiol), synthetic (for example, WIN-55,212-2 and HU-210) and endogenous cannabinoids (for example, anandamide and 2-AG) on cancer cell proliferation and apoptosis were revisited.

In 1998, De Petrocellis et al. investigated the possible antimitogenic effects of AEA on epithelial human breast cancer cell lines EFM-19 and MCF-7. In these models, treatment with sub-micromolar concentration of AEA (as well as of 2-AG or HU-210) significantly inhibited the G1-S transition of mitotic cell cycle. Moreover, AEA inhibited the expression of prolactin receptors, induced downregulation of the brcal gene product [13], and of trk proteins, the high-affinity neurotrophin receptors [14]. The block of the G1-S transition was ascribed to the inhibition of adenylyl cyclase and, consequently of cAMP-protein kinase A pathway and to the activation of MAPK [15]. Cannabinoids prevented the inhibition of RAF1 (caused by protein kinase A-induced Raf phosphorylation) and induced prolonged activation of the RAF1MEK-ERK signalling cascade, leading to downregulation of PRLr and Trk [14]. Mimeault et al. [16] showed that a micromolar concentration of AEA inhibited EGF-induced proliferation of DU145 and PC3 cells (androgen independent human prostate cancer cell lines overexpressed EGF-R) as well as of androgen-stimulated LNCaP, via G1 arrest, and downregulated EGF-R levels. Both phenomena were CB1-mediated. Similar growth arrest and receptor modulation were also reported for prolactin- and nerve growth factor-stimulated DU145 [13,14]. It is important to remark that longer AEA incubation times (5-6 days) were able to induce massive apoptosis in DU145 and PC 3 cells. This effect was mediated by $\mathrm{CB} 1 / 2$ receptors via cellular ceramide accumulation, and was absent in LNCaP cells [16].

The anti-tumoural action of cannabinoids on glioma may be exerted either via the $\mathrm{CB} 1$ or the $\mathrm{CB} 2$ receptor. THC induced apoptosis of $\mathrm{C} 6$ glioma cells by a pathway involving $\mathrm{CB} 1$ receptor, sustained generation of the proapoptotic lipid ceramide and prolonged activation of Rafl/ MEK/ERK cascade. Cannabinoids induced regression of gliomas in vivo; in fact intratumour administration of THC and WIN-55,212-2 induced regression of C6-derived glioma in Wistar rats and in RAG-2deficient mice [17].

Cannabinoid receptors could have a protective role against programmed cell death, as reported in human neuroblastoma and C6 cells, where AEA induced apoptosis, via vanilloid receptors, increasing intracellular calcium concentration, activating COX, releasing cytochrome $c$ and activating caspase 3 [18]. The mechanism through which AEA induces apoptosis in cells expressing both functional cannabinoid and vanilloid receptors is still controversial and might depend on the experimental conditions used.

Initially considered to exert their anti-tumoural actions by proliferation arrest or apoptosis, cannabinoids and their receptors are now emerging as suppressors of angiogenesis and tumour metastatic spreading. Increasing evidence suggests that antitumour effect of cannabinoid-related drugs could be at least in part ascribed to the inhibition of tumour neo-angiogenesis in animal models. The non-psychoactive CB2-agonist cannabinoid JWH-133 inhibited in vitro human umbilical vein endothelial cells (HUVEC) migration and survival [19]; in vivo, JWH-133 treatment of C6 glioma- and grade IV astrocytoma-derived tumours reduced expression levels of proangiogenic factors that destabilize vessel integrity, facilitate vessel sprouting and endothelial cells growth, disrupte the extracellular matrix organization.
Casanova et al. [20] evaluated the potential antiangiogenetic power of cannabinoids in mouse skin carcinoma cell line (PDV-C57) expressing high levels of activated ras and EGF-R and showed that WIN-55,212-2 was able to arrest in vivo the growth of highly malignant PDV-C57 cells-derived tumours. In this model, cannabinoid treatment decreased the expression of proangiogenetic factors VEGF, Ang2 and placental growth factor (PlGF).

Similarly, Met-F-AEA, by inhibiting p21ras activity, prevented the growth of v-Kras- transformed rat thyroid cells both in vitro and in vivo [21]. Furthermore, it inhibited growth of already established tumours by reducing the expression of both VEGF and its receptor Flt1, and upregulating the levels of the cyclin-dependent kinase inhibitor p27kip [22]. Cannabinoids' variable effects on cell migration seem to be dependent on both cellular differentiation levels and specific activation of different receptors. Song and Zhong [23] demonstrated that cannabinoid agonists (HU210, WIN 55212-2, AEA) induced migration of human embrionic kidney 293 cells. The anandamideinduced cell migration was CB1- mediated in human embrionic kidney 293 cells and it was blocked by PD98059 (MAPK inhibitor), suggesting that ERK was crucial for CB1-mediated migration. On the other hand, the antitumour effects of cannabidiol (CBD), a non-psychoactive cannabinoid, could be ascribed, beside to the antiproliferative action on U87 and U373 human glioma cells in vitro and in vivo [24], to inhibition of migration.

Moreover, Met-F-AEA was able to inhibit proliferation of a metastasis-derived thyroid cancer cell line, MPTK-6, more efficaciously than of the primary thyroid cancer-derived TK-6 cells [22]. To test the in vivo effects of Met-F-AEA on induction of metastatic foci, the authors used the Lewis lung carcinoma model of metastatic spreading and demonstrated that Met-F-AEA efficaciously interfered with the formation of lung metastatic nodules by acting on CB1 receptors. In androgen-independent prostate cancer cell lines PC3 and DU145, $2-A G$ reduced invasion through the $\mathrm{CB} 1$-dependent inhibition of adenylyl cyclase, decreasing phospho-kinase A (PKA) activity [25].

\section{Novel therapeutic applications of cannabinoids in cancer disease}

Considering that cannabinoids are able to inhibit the growth of cells transformed in vitro as well as the proliferation of breast tumor cells MCF-7, glioblastoma cells C6 and prostate tumor cells PC-3, the possible therapeutic applications of cannabinoids in cancer disease is strongly supported. Indeed, medical doctors may prescribe THC or certain synthetic as appetite stimulants and vomit inhibitors in patients with AIDS or cancer treated chronically with chemotherapy.

A very interesting study [26] makes a novel use of cannabinoids in the treatment of brain tumors, and it is based on the observations of cannabinoid-induced marked regressions (implying a longer life) and even eradication (implying curation) of glioblastomas in laboratory animals. This study involves a technically simple therapy lacking any significant side effects, and more significantly very effective in the treatment of brain tumors, which as mentioned before cannot be satisfactorily treated nowadays by any other techniques or compounds. The therapy with cannabinoids in the treatment of cerebral tumors involves (intracranial or systematic) administration of (natural of synthetic) cannabinoids to (human or non-human) mammals having cerebral tumors. Activation of the specific receptors of the cannabinoid leads to selective death of the transformed cells. Regression or eradication of the cerebral tumors is achieved without any significant side-effects [26]. 
Another study provides compounds and pharmaceutical formulations thereof that are useful in the treatment, amelioration, and/or prevention of diseases, conditions and/or disorders modulated by a cannabinoid (CB) receptor, especially those modulated by the CB1 or CB2 receptor cell growth related syndromes, dysregulated mammalian cell proliferation, breast cancer cell proliferation, prostrate cancer cell proliferation and the like [27]. The compounds of this study may also be used in conjunction with other pharmaceutical agents for the treatment of the diseases, conditions and/or disorders described herein [27]. This study further provides a method of treating a disease, condition and/or disorder modulated by a cannabinoid receptor (CB), and in particular the $\mathrm{CB} 1$ or $\mathrm{CB} 2$ receptor, in a subject in need thereof by administering to the subject a therapeutically effective amount of a compound or a pharmaceutical composition of the present invention [27].

Another study is based on the discovery that non-psychoactive THC derivatives, such as THC acids, can decrease cell proliferation. Moreover, this effect is not dependent on an increase in the rate of apoptosis, which has been identified as a $\mathrm{CB} 1$ receptor-mediated activity of THC [28]. Accordingly, this study features a method of decreasing cell proliferation in a mammal (e.g., a human) by identifying a mammal in which a decrease in cell proliferation is desirable, and administering to the mammal an amount of a compound of Formula I effective to decrease cell proliferation in the mammal [28]. The methods of the invention provide a new use for non-psychoactivecannabinoid as drugs for the treatment or prophylaxis of a condition or disease characterized by cell proliferations (e.g.,cancer). Because of the low toxicity, nonpsychoactive nature, and low abuse potential of such cannabinoids, the compounds can be used as a dietary supplement (e.g., like a daily vitamin pill) to prevent cancer. In addition, the compounds can be applied topically, e.g., to a skin lesion characterized by undesirable cell proliferation, such as in psoriasis [28].

It has now been found that certain analogs of anandamide are potent inhibitors of transport of anandamide across cell membranes. The inventive analogs do not activate the cannabinoid receptors or inhibit anandamide hydrolysis per se but instead prevent anandamide reuptake thereby prolonging the level of the undegraded anandamide. Previously, cannabinoid drugs were targeted toward cannabinoid receptors and amidase enzymes. The anandamide transport inhibitor of the present invention targets activity of the anandamide transporter [29]. Some of the inventive analogs described herein, and physiologically acceptable salts thereof, have high potential when administered in therapeutically effective amounts for providing a physiological effect useful to treat pain; peripheral pain; glaucoma; epilepsy; nausea such as associated with cancer chemotherapy; cancer. Thus, another aspect of this study is the administration of a therapeutically effective amount of an inventive compound, or a physiologically acceptable salt thereof, to an individual or animal to provide a physiological effect [29].

Some novel tricyclic cannabinoid exhibit fluorescence properties. The fluorescent cannabinoid compounds are typically endogenously fluorescent. Some of these compounds, when administered in a therapeutically effective amount to an individual or animal, result in a sufficiently high level of that compound in the individual or animal to cause a physiological response. The physiological response useful to treat a number of physiological conditions [30]. This study relates generally to cannabinoid compounds. One embodiment of the present invention more particularly relates to cannabinoid compounds exhibiting fluorescence properties, particularly in the ultraviolet-visible wavelength ranges [30]. The inventive compounds described herein, and physiologically acceptable salts thereof, have pharmacological properties when administered in therapeutically effective amounts for providing a physiological response useful to treat nausea associated with cancer chemotherapy [30]. The compound of this study can be administered by a variety of known methods, including, for example, orally, rectally, or by parenteral routes (e.g., intramuscular, intravenous, subcutaneous, nasal or topical). The form in which the compounds are administered will be determined by the route of administration. Such forms include, but are not limited to, capsular and tablet formulations (for oral and rectal administration), liquid formulations (for oral, intravenous, intramuscular, subcutaneous, ocular, intranasal, inhalation-based and transdermal administration) and slow releasing microcarriers (for rectal, intramuscular or intravenous administration). The formulations can also contain a physiologically acceptable vehicle and optional adjuvants, flavorings, colorants and preservatives. Suitable physiologically acceptable vehicles include, for example, saline, sterile water, Ringer's solution and isotonic sodium chloride solutions. The specific dosage level of active ingredient will depend upon a number of factors, including, for example, biological activity of the particular preparation, age, body weight, sex and general health of the individual being treated [30].

Another study relates to the targeting of CB2 cannabinoid receptors as a novel therapy to treat malignant lymphoblastic disease, particularly by adminsitration of active molecules possessing at least some effective CB2 receptor agonist activity to patients sufferring from such disease [31]. Recently, anandamide was shown to inhibit the proliferation of human breast cancer cell lines MCF-7 and EFM-19 in vitro. Also, THC was shown to induce apoptosis in human prostate PC-3 cells and in C6 glioma cells in culture. THC-induced apoptosis involved cannabinoid receptor-dependent or -independent pathways. Such studies have triggered interest in targeting cannabinoid receptors in vivo to induce apoptosis in transformed cells. To this end, cannabinoids were shown recently to inhibit the growth of C6 glioma cells in vivo [31]

The present studies have noted that cells of the immune system express high levels of CB2 receptors which they considered might be implicated in induction of apoptosis in normal or transformed immune cells. By using both murine and human leukemia and lymphoma lines as well as primary acute lymphoblastic leukemia (ALL) cells they have demonstrated that ligation of $\mathrm{CB} 2$ receptors can induce apoptosis in a wide range of cancers of immune-cell origin. Furthermore, they demonstrate that TEC can inhibit the growth of murine lymphoma cells in vivo by inducing apoptosis and, in test experiments, completely cure approximately $25 \%$ of the mice bearing that tumor. Current data suggest that CB2 agonists that are devoid of psychotropic effects may constitute a novel and effective modality to treat malignancies of the immune system [31]. These studies have particularly found that exposure of murine tumors EL-4, LSA, and P815 to delta-9tetrahydrocannabinol (THC) in vitro led to a significant reduction in cell viability and an increase in apoptosis. Exposure of BL-4 tumor cells to the synthetic cannabinoid HU-210 and the endogenous cannabinoid anandamide lcd to significant induction of apoptosis, whereas exposure to WINS55212 was not effective. Treatment of EL-4 tumor bearing mice with THC in vivo led to a significant reduction in tumor load, increase in tumor-cell apoptosis, and increase in survival of tumor-bearing mice [31]. These studies have examined of a number of human leukemia and lymphoma cell lines, including Jurkat, Molt-4, and Sup-T1, and have determined that they expressed CB2 but not CB1 receptors. These human tumor cells were also susceptible to apoptosis induced by THC, HU-210, anandamide, and the CB2-selective agonist JWH-015. This effect was mediated at least in part through the $\mathrm{CB} 2$ receptors because pretreatment with the CB2 antagonist SR144528 partially reversed 
the THC-induced apoptosis. Culture of primary acute lymphoblastic leukemia cells with THC in vitro reduced cell viability and induced apoptosis. Thus CB2 cannabinoid receptors expressed on malignancies of the immune system are capable of serving as potential targets for the induction of apoptosis. CB2 agonists lack psychotropic effects, they can serve as novel anticancer agents to selectively target and kill tumors of immune origin. The present studies have demonstrated that THC and other cannabinoids can induce apoptosis in murine and human leukemia and lymphoma cell lines as well as primary ALL cells. The human tumor-cell lines screened expressed CB2 but not $\mathrm{CB} 1$ receptors, whereas the murine tumors expressed both $\mathrm{CB} 1$ and $\mathrm{CB} 2$ receptors [31]. Ligation of $\mathrm{CB} 2$ receptors is sufficient to induce apoptosis inasmuch as $\mathrm{CB} 2$-selective agonists can induce apoptosis in tumor cells. THC-induced apoptosis in human tumor-cell lines is now shown to be reversed by CB2 antagonists. THC was effective not only in vitro but also in vivo, as demonstrated by its ability to induce apoptosis and decrease the tumor load. Moreover, THC treatment could cure approximately $25 \%$ of the mice bearing a syngeneic tumor. Thus targeting CB2 receptors on tumor cells of immune origin provides a novel and relatively non-toxic approach to treating such cancers [31].

Another study relates to methods and compositions for treating cancer. More particularly, the study provides cannabidiol derivatives and compositions thereof [32]. Previous studies demonstrated that the helix-loop-helix protein Id-1, an inhibitor of basic helix-loop-helix (bHLH) transcription factors, plays a crucial role during breast cancer progression. Id-1 stimulated proliferation, migration and invasion in breast cancer cells. Moreover, targeting Id-1 expression partially in breast cancer cells reduced invasion and breast cancer metastasis in vitro and in preclinical animal models. The disclosure shows that Id-1 is a target for therapy approaches, and that inhibiting Id-1 expression and/or activity provides a mechanism for treating patients with breast cancer. This approach may be highly effective and safe in advanced breast cancer patients, given (1) the relationship between high Id-1 expression levels and aggressive breast cancer cell behaviors; (2) partial reduction in Id-1 activity can achieve significant outcomes; and (3) Id-1 expression is low in normal adult tissues, thereby eliminating unwanted toxicities generally associated with currently available therapeutic modalities [32]. Id-1 protein plays a key role in the malignant progression of many aggressive and invasive human cancer such as: leukemia, melanoma, hepatocellular carcinoma, colorectal adenocarcinoma, pancreatic cancer, lung cancer, kidney cancer, medullary thyroid cancer, papillary thyroid cancer, astrocytic tumor, neuroblastoma, Ewing's sarcoma, ovarian tumor, cervical cancer, endometrial carcinoma, breast cancer, prostate cancer, malignant seminoma, and squamous cell carcinomas, such as esophageal cancer, and head and neck cancer. Accordingly, Id-1 associated cell proliferative disorders include, but are not limited to, Leukemia, Melanoma, Squamous cell carcinoma (SCC) (e.g., head and neck, esophageal, and oral cavity), Hepatocellular carcinoma, Colorectal adenocarcinoma, Pancreatic cancer, Lung cancer, Kidney cancer, Medullary thyroid cancer, Papillary thyroid cancer, Astrocytic tumor, Neuroblastoma, Ewing's sarcoma, Ovarian tumor, Cervical cancer, Endometrial carcinoma, Breast cancer, Prostate cancer, and Malignant seminoma [32]. Approaches for targeting Id-1 expression include gene therapy using antisense oligonucleotide, siRNA, non-viral or viral plasmid-based strategies. In addition, the development of new strategies to modulate Id-1 expression/functional activity include the identification of small molecules that modulate the activity of Id-1. A range of small molecules that target the molecular pathology of cancer are now being developed, and a significant number of them are being tested in ongoing human clinical trials. The disclosure demonstrates that cannabidiol (CBD) and CBD derivatives are inhibitors of Id-1. The use of $\mathrm{CBD}$, and derivatives thereof, represents a novel strategy for the treatment of cancer [32]. A variety of chemical classes of cannabinoids are useful in the methods provided herein including cannabinoids structurally related to THC, aminoalkylindoles, the eicosanoids related to the endocannabinoids, 1,5-diarylpyrazoles, quinolines and arylsulphonamides and additional compounds that do not fall into these standard classes but bind to cannabinoid receptors [32]. Data provided herein indicates that $\mathrm{CBD}$ and derivatives thereof that act as Id-1 inhibitor effectively inhibit genotypic and phenotypic changes that allow aggressive breast cancers to proliferate, invade and metastasize [32]. Since CBD inhibits Id-1 expression in aggressive breast cancer, the disclosure also provides a rational drug design strategy and compounds obtained there from as potent and efficacious analogs. The disclosure demonstrates that the opened tetrahydropyran ring in CBD and aliphatic side chain of CBD are key pharmacophores involved in the inhibition of Id-1, alterations of these functional groups allow one to improve both the potency and efficacy of the parent compound, CBD [32]. Moreover, reducing Id-1 expression with cannabinoids provides a therapeutic strategy for the treatment of additional aggressive cancers since Id-1 expression was found to be up-regulated during the progression of almost all types of solid tumors investigated [32]. Accordingly, provided herein are methods for modulating the activity of a metastatic cell by regulating the activity of a target Id-1 using a CBD or CBD derivative. Methods can also include "regulating the activity of a target Id-1" includes: 1) mechanisms for modulating endogenous nucleic acid sequences that encode a target Id-1 such that Id-1 polypeptide levels are decreased in a cell; 2) introducing exogenous nucleic acid sequences that inhibit Id-1 expression in a cell; 3 ) increasing the turnover rate of endogenous Id-1 polypeptides such that Id-1 polypeptide levels are decreased in a cell [32].

\section{Conclusions}

The endocannabinoid system is an almost ubiquitous signalling system involved in the control of cell fate. Recent studieshaveinvestigated the possibility that drugs targeting the endocannabinoid system might be used to retard or block cancer growth. The endocannabinoids have been shown to inhibit the growth of tumour cells in culture and animal models by modulating key cell signalling pathways $[33,34]$. In previous studies, we reported that stimulation of cannabinoid CB1 receptors by the metabolically stable endocannabinoid analogue Met-F-AEA inhibits apex ras activity, prevents proliferation of $\mathrm{v}$-K-ras-transformed rat thyroid cells both in vitro and in vivo and is also able to block the growth of already established tumours [21]. Indeed, our very recent data show that Met-F-AEA significantly inhibits, in tumours as well as in transformed cells, the expression of the vascular endothelial growth factor (VEGF), an angiogenetic factor known to be up-regulated by p21ras, as well as of one of its receptors, flt-1/VEGFR-1. The levels of the cyclin-dependent kinase inhibitor p27(kip1), which is downregulated by $\mathrm{p} 21 \mathrm{ras}$, were instead increased by Met-F-AEA. All these effects were antagonized by the selective $\mathrm{CB} 1$ receptor antagonist SR141716A. Met-F-AEA inhibited in vitro the growth of a metastasisderived thyroid cancer cell line more potently than a primary cancer cell line. It has been shown that Met-F-AEA significantly reduced the number and size of metastatic nodes in an animal model of metastatic spreading (formation of lung nodules after inoculation of 3LL cells), in a way antagonized by SR141716A [22]. Therefore, the present review indicated that cannabinoids exert their anti-cancer effects in a number of ways and in a variety of tissues.

- Triggering cell death, through a mechanism called apoptosis

- Stopping cells from dividing

- Preventing new blood vessels from growing into tumours 
- Reducing the chances of cancer cells spreading through the body, by stopping cells from moving or invading neighbouring tissue

- Speeding up the cell's internal 'waste disposal machine' - a process known as autophagy - which can lead to cell death

Furthermore, the novel therapeutic application of cannabinoids in cancer disease, described here, strongly support the idea that cannabinoids may induce benefical effect in cancer treatment.

\section{References}

1. Adams IB, Martin BR (1996) Cannabis: pharmacology and toxicology in animals and humans. Addiction 91: 1585-1614

2. Mechoulam R(1986) Cannabis as Therapeutic Agent (ed. Mechoulam, R.) 1-19 CRC Press Roca Ranton.

3. Williamson EM, Evans FJ (2000) Cannabinoids in clinical practice. Drugs 60: 13031314. [Crossref]

4. Mechoulam R, Gaoni Y (1967) Recent advances in the chemistry of hashish. Fortschr Chem Org Naturst 25: 175-213. [Crossref]

5. Matsuda LA, Lolait SJ, Brownstein MJ, Young AC, Bonner TI (1990) Structure of a cannabinoid receptor and functional expression of the cloned cDNA. Nature 346: 561-564

6. Munro S, Thomas KL, Abu-Shaar M (1993) Molecular characterization of a peripheral receptor for cannabinoids. Nature 365: 61-65

7. Howlett AC (2005) Cannabinoid receptor signaling. Handb Exp Pharmacol : 53-79. [Crossref]

8. Howlett AC (2002) The cannabinoid receptors. Prostaglandins Other Lipid Mediat 69: 619-631.

9. Herkenham M, Lynn AB, Johnson MR, Melvin LS, de Costa BR, et al. (1991) Characterization and localization of cannabinoid receptors in rat brain: a quantitative in vitro autoradiographic study. $J$ Neurosci 11: 563-583

10. Porter AC1, Felder CC (2001) The endocannabinoid nervous system: unique opportunities for therapeutic intervention. Pharmacol Ther 90: 45-60. [Crossref]

11. Begg M, Pacher P, Batkai S (2005) Evidence for novel cannabinoid receptors. Pharmacol Ther 106:133-145

12. Howlett AC, Breivogel CS, Childers SR, Deadwyler SA, Hampson RE, et al. (2004) Cannabinoid physiology and pharmacology: 30 years of progress. Neuropharmacology 47: $345-358$

13. De Petrocellis L, Melck D, Palmisano A (1998) The endogenous cannabinoid anandamide inhibits human breast cancer cell proliferation. Proc Natl Acad Sci 95: $8375-8380$

14. Melck D, De Petrocellis L, Orlando P (2000) Suppression of nerve growth factor Trk receptor and prolactin receptors by endocannabinoids leads to inhibition of human breast and prostate cancer cell proliferation. Endocrinology 141: 118-126

15. Melck D, Rueda D, Galve Ropher I, De Petrocellis L, Guzman M, et al. (1999) Involvement of the cAMP/protein kinase A pathway and of mitogen-activated protein kinase in the anti-proliferative effects of anandamide in human breast cancer cells. FEBS Lett 463:235-240
16. Mimeault M, Pommery N, Wattez N, Bailly C, Henichart JP (2003) Anti-proliferative and apoptotic effects of anandamide in human prostatic cancer cell lines: implication of epidermal growth factor receptor down-regulation and ceramide production. Prostate 56:1-12

17. Galve-Roperh I, Sanchez C, Cortes ML, Del Pulgar TG, Izquierdo M, et al. (2000) Antitumoural action of cannabinoids: involvement of sustained ceramide accumulation and extracellular signal-regulated kinase activation. Nat Med 6: 313-319

18. Maccarrone M, Lorenzon T, Bari M, Melino G, Finazzi-Agrò A (2000) Anandamide induces apoptosis in human cells via vanilloid receptors. Evidence for a protective role of cannabinoid receptors. J Biol Chem 275: 31938-31945

19. Blázquez C, Casanova ML, Planas A, Gómez Del Pulgar T, Villanueva C, et al. (2003) Inhibition of tumor angiogenesis by cannabinoids. FASEB J 17: 529-531. [Crossref]

20. Casanova ML, Blázquez C, Martínez-Palacio J, Villanueva C, Fernández-Aceñero MJ, et al. (2003) Inhibition of skin tumor growth and angiogenesis in vivo by activation of cannabinoid receptors. J Clin Invest 111: 43-50. [Crossref]

21. Bifulco M, Laezza C, Portella G (2001) Control by the endogenous cannabinoid system of ras oncogene dependent tumour growth. FASEB J 15:2745-2747

22. Portella G, Laezza C, Laccetti P, De Petrocellis L, Di Marzo V, et al. (2003) Inhibitory effects of cannabinoid $\mathrm{CB} 1$ receptor stimulation on tumour growth and metastatic spreading: actions on signals involved in angiogenesis and metastasis. FASEB $J$ 17:1771-1773

23. Song ZH, Zhong M (2000) CB1 cannabinoid receptor-mediated cell migration. $J$ Pharmacol Exp Ther 294: 204-209

24. Massi P, Vaccani A, Ceruti S, Colombo A, Abbracchio MP, et al. (2004) Antitumour effects of cannabidiol, a nonpsychoactive cannabinoid, on human glioma cell lines. $J$ Pharmacol Exp Ther 308: 838-845

25. Nithipatikom K, Endsley MP, Isbell MA (2004) 2-Arachidonoylglycerol: a novel inhibitor of androgen-independent prostate cancer cell invasion. Cancer Res 64 : 8826-8830

26. Guzman P, Manuel SG, Cristina GR (2004) Therapy with cannabinoid compounds for the treatment of brain tumors United States Patent Application.

27. Muthuppalaniappan M; Balasubramanian G, Gullapalli S, Joshi NK; Narayanan S (2008) Novel Cannabinoid Receptor Ligands, Pharmaceutical Compositions Containing Them, and Process For Their Preparation, United States Patent Application 2008/0234259.

28. Burstein A, Summer R, Lawrence Z, Robert B (2004) Cannabinoid drugs United States Patent Application 2004/0225011.

29. Makriyannis A, Goutopoulos A, Li C (2009) Inhibitors of the anandamide transporter United States Patent 7589220

30. Makriyannis A, Lu D, Lai X (2007) Keto cannabinoids with therapeutic indications United States Patent 7183313.

31. Nagarkatti L, Nagarkatti P, Mckallip R; Lombard C, Ryu S (2004) Treatment of neoplasia.United States Patent Application 2004/0259936.

32. Guzmán M (2003) Cannabinoids: potential anticancer agents. Nat Rev Cancer 3: 745755. [Crossref]

Copyright: (C2017 Milano W. This is an open-access article distributed under the terms of the Creative Commons Attribution License, which permits unrestricted use, distribution, and reproduction in any medium, provided the original author and source are credited. 